\title{
¿Socialización o individuación? Dos enfoques para examinar la inducción de los nuevos trabajadores
}

Socialization or individuation?

Two approaches to review the induction of new employees

Socialisation ou individuation? Deux approches pour examiner l’induction des nouveaux travailleurs

\section{Resumen}

Héctor L. Bermúdez Restrepo hector-leonel.bermudez@hec.ca École des Hautes Études commerciales - HEC Montreal, Canadá.

Sociólogo. Magíster en Ciencias de la Administración. Chargé de cours Sociología de la empresa, Service de l'enseignement du management HEC Montréal.

Artículo de revisión Según Clasificación Colciencias

Fecha de recepción: $18 / 07 / 2012$

Fecha de corrección: $14 / 11 / 2012$

Fecha de aprobación: $10 / 12 / 2012$
En general, los seres humanos, al comenzar una experiencia laboral, se someten a un ajuste biográfico de mayor o menor intensidad. Examinar tal experiencia es una oportunidad para los especialistas, tanto del management en general, como de la gestión humana en particular. En este artículo se propone un marco de referencia para estudiar la etapa de orientación de los nuevos trabajadores al ingresar a la empresa. Para hacerlo, se acude a ciertos recursos teóricos de dos enfoques sociológicos diferentes pero complementarios: la socialización y la individuación. Se descubrió que la tradición epistemológica utilizada tanto por el management en general, como por la gestión humana en particular, privilegia una de estas dos versiones sociológicas y, en general, desconoce la otra. De hecho, se confunde un proceso administrativo como lo es la inducción general a la empresa, con un fenómeno sociológico de gran complejidad: la configuración social del trabajador contemporáneo.

Palabras clave: gestión humana, socialización organizacional, individuación, individualización. 


\section{Socialization or individuation? Two approaches to review the induction of new employees}

\section{¿Socialización o individuación? \\ Dos enfoques para examinar la inducción de los nuevos trabajadores}

\section{Socialisation ou individuation? Deux approches pour examiner l'induction des nouveaux travailleurs}

\section{Abstract}

Upon starting a work experience, humans are subjected to a biographical adjustment of lesser or greater intensity. Examining this experience is an opportunity for specialists in management and human management. This article seeks to reflect upon a framework to study the orientation stage of new workers joining the company. To do this, we turn to some theoretical resources of two different but complementary sociological approaches: socialization and individuation. It was found that management and human management favor one of these two sociological versions, but ignores the other. In fact, it confuses an administrative process like the general induction to the company with a highly complex sociological phenomenon: the social setting of the contemporary worker.

Keywords: personnel management, organizational socialization, individuation, individualization.
Socialisation ou individuation? Deux approches pour examiner $l^{\prime}$ induction des nouveaux travailleurs

¿Socialización o individuación?

Dos enfoques para examinar la inducción de los nuevos trabajadores

\section{Socialization or individuation?}

Two approaches to review the induction of new employees

\section{Résumée}

En général, les êtres humains, au moment de commencer une nouvelle expérience de travail, se soumettent à un ajustement biographique de majeure ou mineure intensité. Examiner telle expérience c'est une opportunité pour les spécialistes, tant du management en général, que pour la gestion humaine en particulier. Cet article propose un cadre de référence pour étudier l'étape d'orientation des nouveaux travailleurs au moment de leur entrée à l'entreprise. Pour cela, il faut faire recours à certaines ressources théoriques de deux approches sociologiques différentes mais complémentaires : la socialisation et l'individuation. On a découvert que la tradition épistémologique utilisée tant par le management général, que par la gestion humaine en particulier, privilégie une de ces versions sociologiques et, en général, méconnait l'autre. En effet, on confond un processus administratif tel que l'induction générale à l'entreprise, avec un phénomène sociologique de grande complexité : la configuration sociale du travailleur contemporain.

Mots clef: gestion humaine, socialisation organisationnelle, Individuation, Individualisation. 


\title{
¿Socialización o individuación? Dos enfoques para examinar la inducción de los nuevos trabajadores
}

\author{
Ulysse se dressa sur ses jambes et, examinant ce qui était sa propre terre, s'écria plein de tristes- \\ se : "Malheur ! Où ai-je à présent atterri ? Qu'est-ce que je fais ici ? "
}

\section{Introducción}

Desde hace más de cuarenta años, la literatura especializada en gestión humana insiste en asociar la etapa de llegada de los nuevos trabajadores a la empresa con un proceso de socialización (Schein, 1968, 1971; Van Maanen, 1976; Van Maanen y Schein, 1979). Este tipo de premisas, poco problematizadas, hace pertinente la pregunta sobre aquello que les sucede a las personas cuando comienzan una nueva experiencia de trabajo en la empresa. Sin embargo, para reflexionar sobre la actualidad de este interrogante, es preciso hacer dos delimitaciones previas: ¿De qué tipo de empresa se trata? ¿Qué se entiende exactamente por etapa de llegada? Para definir la primera de estas dos preguntas, durante el desarrollo del artículo se hará evidente que se trata, sobre todo de las empresas regidas por la lógica de la gran corporación privada contemporánea; este tipo de organizaciones utiliza, "modelos estratégicos de gestión humana" (Bermúdez, 2010; Bustamante y Bermúdez, 2010)ํ․ Esto último permite, comenzar a responder a la segunda pregunta, porque en este tipo de modelos de gestión, una vez hecha la selección de personal, la dirección se debe encargar de administrar la "etapa de orientación" del nuevo trabajador (Notais, 2010, 2009; Perrot, 2009, 2008; Bargues, 2008, 2006; Boussaguet, 2007, 2005; St-Onge et al., 2004; Riordan et al., 2001).

Se pudo constatar en la literatura de la gestión humana en particular, y en muchos autores de la sociología de la empresa, que durante la etapa de llegada, al trabajador se le considera en un proceso de socialización. Algunos nombran tal proceso como "socialización organizacional" o "inducción general", y otros lo llaman "socialización profesional". Inspirados en una de las ideas fundadoras de Hawthorne, donde la organización se debe comprender como un "sistema de acción cooperativa" (Barnard, 1938; Roethlisberger y Dickson, 1939), algunos autores se refieren a la empresa como a un "sistema social establecido" (Schein, 1982) o un "sistema de acción organizada" (Crozier y Friedberg, 1977). Por su parte, un autor como Giddens (1991), comprende a la actividad laboral como a una "agencia de socialización”. Así, para analizar la llegada del trabajador a la empresa, es común encontrar la siguiente categorización: a) la organización se considera como un dispositivo de socialización, y b) el trabajador es comprendido como un individuo que está siendo socializado.

Sin embargo, este enfoque es incompleto, porque privilegia una estrategia de análisis sociológico que deja de lado otras perspectivas teóricas y metodológicas. En otras palabras: el enfoque mencionado favorece la figura del "personaje social" (Martuccelli, 2002). Lo anterior quiere decir que, en aquella perspectiva, el individuo no es más que "la otra cara del sistema social", lo que obliga a sospechar que se puede estar utilizando una sociología en desuso:

Antes, el individuo era ante todo tomado de una manera o de otra, por la idea de la socialización, concebida como un proceso de apropiación progresiva de lo social por parte del actor, gracias al cual él se dotaba de competencias necesarias para vivir en sociedad (Martuccelli, 2002, pp. 28-29) ${ }^{2}$.

No obstante, la sociología contemporánea indica que actualmente, estamos en una nueva sociedad, en una "modernidad avanzada" (Giddens, 1993), en una "sociedad ultra-contemporánea” (Gauchet, 1998), o en los "tiempos hipermodernos" (Lipovetsky, 2004), etc. Hoy, se asiste al advenimiento de un indivi-

1. Es preciso señalar que estos modelos no son exclusivos de la gran empresa privada; la influencia de la lógica del management y sus modelos de gestión, se pueden observar también, tanto en la pequeña empresa, como en la gestión pública (Gaulejac, 2005), principalmente pp. 25-108 y Aktouf (2008, 2002).

2. En todo el artículo, traducción libre de los textos consultados en francés. 
duo "incierto" o "fatigado" (Ehrenberg, 1998, 1995), un individuo "desafiliado" (Castel, 1997), o un hombre "improvisador" (Conninck y Guillot, 2007) ${ }^{3}$. Así, para comprender las problemáticas sociales actuales, es preciso utilizar una "nueva" sociología. Más concretamente, para lograr un análisis del ser humano como trabajador de esta contemporaneidad, es necesario completar el enfoque sociológico utilizado hasta el presente por los autores de la gestión humana. En este escrito se propone complementar la perspectiva de la socialización con otra estrategia de análisis sociológico: aquella de la individuación.

Así, el lector encontrará en este artículo los principales aspectos sobre la perspectiva de las empresas que utilizan modelos estratégicos de gestión humana y que justifican hablar de gestionar la "etapa de llegada". Luego se muestran algunas observaciones metodológicas acerca de la complementariedad de los dos enfoques, el de la socialización y el de la individuación. También se presenta la descripción de cada uno de los dos. Por último, se señala la importancia de articular e integrar esta conceptualización antes de proceder a la validación de campo. Aquí no se pueden presentar conclusiones a la manera de los hallazgos del trabajo empírico, sino como ciertas conquistas teóricas y metodológicas logradas en la revisión de la literatura especializada, que atraviesan todo el artículo y que pretenden brindarse a los estudiosos de la gestión humana para el examen de las dinámicas complejas de los trabajadores al arribar a los escenarios organizacionales contemporáneos.

\section{La perspectiva de la empresa}

Numerosas investigaciones permiten asegurar que en general, las empresas contemporáneas están cada día más de acuerdo en utilizar "modelos estratégicos de gestión" para llevar a cabo la administración del personal (Bermúdez, 2010, 2009; Bustamante, 2006; Bayad, Arcand y Allani, 2004; Sekiou et al., 2001; Wils, Labelle, Guérin, 1989) 4 . Estos modelos dan mucha importancia a la etapa de recibimiento de los nuevos trabajadores. Para hacer esto, tanto los ejecutivos como los académicos del dominio de la gestión humana proponen utilizar unos procesos diseñados, aplicados y evaluados rigurosamente. Esta etapa de orientación es comprendida como una socialización del nuevo trabajador a la organización y se utiliza ese término para nombrarla. Cuando se consulta a los ejecutivos y a los responsables de la gestión del personal en las organizaciones, en general, coinciden en apreciar mucho esta etapa y están de acuerdo en que es un proceso de suma importancia. Así, destacan que hay que hacerlo para adaptar y ajustar al nuevo trabajador a la empresa, para minimizar sus temores y ansiedades y desarrollar rápidamente el sentido de gratitud y de pertenencia con el fin de lograr su contribución inmediata y efectiva.

De igual manera, muchos autores de este campo de estudio, consideran que el "empleado" deberá aceptar la realidad y adherirse a un sistema de normas que ya está establecido por sus "superiores". Se pudo constatar que estos académicos piensan que tal etapa de recibimiento es muy importante para los intereses de la empresa y priorizan, como ellos mismos afirman, por "el tiempo y el dinero de la organización". Sin embargo, a pesar de sus esfuerzos, desconocen muchos aspectos netamente sociales. En general, proponen la "integración" del nuevo trabajador por la vía del adiestramiento (St-Onge et al., 2004, p. 228). Al igual que los ejecutivos, estos autores de textos reconocidos, prestan su atención a la etapa de orientación y la definen como la "socialización del nuevo empleado", nombran a los trabajadores como "valiosos recursos" y consideran al proceso como "fundamental" (Schein, 1982; Cane, 1997; Dolan, Schuler y Valle, 1999; Gómez-Mejía, Balkin y Cardy, 1999; Koontz y Weihrich 1994; Cascio, Thacker y Blais, 1999; Chiavenato, 2002).

Estos autores afirman que el nuevo trabajador debe ser introducido a su puesto para que se convierta, lo más pronto posible, en "una parte

3. En cuanto al análisis del actual contexto social, antes de preguntarse por el trabajador como individuo contemporáneo, hay que hacer una advertencia de orden epistemológico. Es preciso diferenciar las corrientes que parten de la premisa de una ruptura con la modernidad, de aquellas que piensan que estamos en una cierta continuidad con la modernidad. De acuerdo con Soussi (2009), por ejemplo, algunas de estas perspectivas son, sin lugar a dudas, irreconciliables entre sí (la de Lipovetsky con respecto de la de Giddens, es un claro ejemplo) (Bonny, 1998 y 2004).

4. Aunque el concepto de "modelo estratégico de gestión humana" es reciente, la propuesta de una "interacción directa" entre la estrategia de la empresa y la gestión de personal no es nueva. Al respecto Wils et al. (1989, pp. 354-355) advierten: "La idea ha sido presentada al principio de los años 70 (Fox, 1973). Más tarde por Walker (1978), luego por Fombrun, Tichy y Devanna, 1984; y por Bélanger et al., 1988”. 
productiva de la fuerza de trabajo". Entonces, consideran que es muy importante brindar la orientación para que él desarrolle el sentido de "pertenencia y compromiso por la empresa" (Cane, 1997, p. 68). Sostienen también, que la organización tiene necesidad de "recursos humanos" que compartan los diferentes objetivos estratégicos y que se comprometan a apoyarlos, por lo cual, el proceso de orientación general, resulta para ello, el instrumento perfecto. Por su parte, los ejecutivos también están de acuerdo. Consideran muy importante la etapa de llegada del nuevo trabajador, definen esta etapa como la socialización y diseñan un proceso al que llaman la "inducción general":

La inducción es el programa de recibimiento del nuevo empleado. Este programa puede llamarse también "orientación”, "integración”, "socialización”. Además de recibir y orientar a las personas que se vinculan, la inducción general también es muy importante porque, cuando un individuo nuevo llega a la organización, es el primer contacto de éste con la empresa, y las primeras impresiones causadas son fundamentales en el desarrollo laboral del individuo dentro de ella. Además es una oportunidad para transmitirle información básica y coherente sobre la empresa, reafirmando con esto las bases de la cultura organizacional (Departamento de Formación, Almacenes EXITO, 2001, s.p).

En resumen, tanto los ejecutivos como los autores de textos especializados en gestión humana, consideran muy importante la etapa de recibimiento de los nuevos trabajadores y están de acuerdo en asociarla con una socialización. De hecho, como ya se advirtió, nombran el proceso de orientación con este nombre, consideran este período como una etapa de "adaptación" y diseñan procesos administrativos con esta intención. Hay una constante en los ejemplos estudiados en esta investigación: las empresas animan la adopción de las normas, la habituación, la obediencia. Esperan que el nuevo trabajador se adapte al escenario, que se ajuste a un mundo enteramente dado.

Sin embargo, los nuevos trabajadores no son sujetos pasivos de adaptación. Según la perspectiva de los responsables de dirigir las empresas, ellos han sido contratados para contribuir a los objetivos de la organización a la que llegan. Pero esta visión es bastante reduccionista. Hay que preguntarse por la otra cara de la moneda: ¿Cuál es la perspectiva de los trabajadores? ¿Acaso es la misma de las empresas? Ya se vio que los ejecutivos de la gestión humana en las organizaciones requieren minimizar los temores y las ansiedades de los trabajadores para encontrar rápidamente el sentido de gratitud y de pertenencia y así obtener su contribución inmediata y efectiva. Ellos piensan que los aportes de los trabajadores serían probablemente más auténticos, más efectivos, o menos problemáticos, en la medida en que las angustias ocasionadas por lo desconocido sean equilibradas por las ilusiones de llegar a un colectivo que les recibe y que favorece sus expectativas.

Precisamente, estas angustias y estas ilusiones pueden brindar pistas muy importantes para comprender las dinámicas complejas que subyacen en los episodios biográficos que están viviendo los nuevos trabajadores que ingresan: ¿Cómo las experimenta el nuevo trabajador al llegar a la organización? ¿Qué nivel de conciencia tiene él de su proceso de "integración" a la nueva empresa? ¿Qué sentido tiene esto para él? ¿A qué le teme y qué desea? ¿Qué lo anima y que lo inhibe? ¿En qué tipo de individuo está constituyéndose? Sin duda, el nuevo trabajador puede estar siendo sometido a una profunda contradicción existencial, de la que no siempre tiene conciencia. Es preciso pues, buscar las pistas justamente en la manera como el trabajador dice vivir su etapa de llegada.

Se insiste entonces que si se trata de analizar el comienzo del nuevo trabajador en la empresa, se requiere examinar la etapa de recibimiento según dos enfoques de análisis sociológico diferentes pero complementarios. De una parte, privilegiando el proceso de fabricación identitario del actor, es decir, su socialización; pero, del otro lado, privilegiando la estrategia de análisis de la individuación, es decir, preguntándose: ¿Qué tipo de individuo está siendo estructuralmente fabricado en la sociedad actual?

\section{Advertencia metodológica: ¿socialización o individuación?}

Si se parte de la premisa de que el nuevo trabajador es un individuo que llega a una comunidad que le precede, para examinar su integración a la empresa, se puede pensar que, en efecto, tal integración se trata de una especie de socialización. Así, se puede tomar, metodológicamente hablando, al individuo "por separado" y preguntarse cómo sucede este proceso de socialización. 
Además, si se toma a la empresa como un lugar "dado", como un escenario "establecido", se estaría apuntando entonces hacia la noción de socialización organizacional, idea desarrollada por Schein, desde 1968. Pero, si se toma a la organización como una manera particular de organización social del trabajo, se apunta entonces a la noción de socialización profesional (Dubar, 2006a). Sin embargo, como se verá, en los dos casos, se está en un tipo de interpretación de la realidad que privilegia la ilusión del orden social.

Para examinar la noción de socialización, en la presente investigación, se acudió a Dubar (2007, 2006a, 2006b). Esto gracias a que, para proponer su "teoría sobre la socialización" él llevó a cabo una delimitación, que resulta de suma utilidad para el estudio de la etapa de llegada de los trabajadores a la empresa. Este autor pretende grosso modo, "suministrar unos instrumentos de análisis, unos marcos teóricos y unos resultados empíricos para comprender la dinámica en curso de la socialización profesional y las identidades sociales" (Dubar, 2006a). Para este autor, es fundamental penetrar en las identidades profesionales. Sin embargo, el objetivo de nuestro estudio es otro. La intención aquí es la de caracterizar cómo la sociología ha comprendido la socialización, para comparar la etapa de llegada de los nuevos trabajadores a la empresa, con este proceso de socialización. Así, se acude a las ideas de Dubar en relación con las "grandes teorías" sobre el análisis de este proceso, y sobre las bases de su propia reactualización de esta noción, para mostrarla como estrategia de análisis sociológico (Tabla 1).

\begin{tabular}{|c|c|}
\hline Autor & Nociones clave \\
\hline Jean Piaget & Asimilación; acomodación \\
\hline Pierre Bourdieu & Habitus; campo social \\
\hline George H. Mead & $\begin{array}{l}\text { Construcción de un si (Self) en la } \\
\text { relación con el otro }\end{array}$ \\
\hline Robert K. Merton & Socialización anticipatoria \\
\hline $\begin{array}{l}\text { Peter Berger \& } \\
\text { Thomas Luckmann }\end{array}$ & $\begin{array}{l}\text { Construcción social de la realidad; } \\
\text { socialización secundaria }\end{array}$ \\
\hline Jürgen Habermas & $\begin{array}{l}\text { Exteriorización de lo subjetivo e } \\
\text { interiorización de lo objetivo }\end{array}$ \\
\hline Max Weber & $\begin{array}{l}\text { Socialización comunitaria; } \\
\text { socialización societaria }\end{array}$ \\
\hline Claude Dubar & $\begin{array}{l}\text { La socialización como articulación de } \\
\text { dos procesos identitarios } \\
\text { heterogéneos }\end{array}$ \\
\hline & Fuente: Bermúdez, H. L. (2012). \\
\hline
\end{tabular}

Sin embargo, tal como se anunció desde el principio del artículo, aquí se propone complementar esta estrategia de análisis de la socialización con otro enfoque: se trata del proceso de individuación. Tal enfoque pretende dar cuenta de la manera cómo se configuran los individuos en la sociedad, es decir, trata del "proceso de constitución de los individuos", según los términos de Martuccelli (2009). La génesis de la teorización de esta estrategia de análisis, puede hallarse en la tesis doctoral de Simondon "La individuación a la luz de las nociones de forma y de información", defendida en 1958 (2009); sin embargo, en el contexto de la presente investigación, se acudió fundamentalmente a Martuccelli (2009, 2005, 2002), autor que no se apoya en las ideas de Simondon, sino en la sociología de los individuos de Elías (1991), y sobre algunas premisas de Beck (1986), entre otros. También, los orígenes de esta estrategia de análisis sociológico pueden cotejarse con ciertas ideas fundadoras acerca del individuo de Weber $(2002,1965)$ y de Simmel (1989), publicadas a principios del siglo $X^{5}$.

Para aplicar el proceso de individuación como "principio de unidad analítica de las sociedades contemporáneas", Martuccelli propone su articulación con un operador analítico, las pruebas, y con una herramienta heurística, los retratos. Pero, hay que insistir que no se trata de reemplazar el estudio de la socialización por el de la individuación, sino de complementarlos. Así, de la misma manera que se dijo qué se hará con la socialización, se reitera entonces, que, de lo que se trata aquí, es de caracterizar cómo la sociología comprende la individuación, para comparar la etapa de llegada de los nuevos trabajadores a la empresa, con tal proceso de individuación.

Se propone entonces, un esquema con dos grandes ejes para examinar la mencionada etapa de llegada de los nuevos trabajadores: de una parte el enfoque de la socialización y de otra, la perspectiva de la individuación (Tabla 3).

\section{Una estrategia de análisis: la socialización}

Durkheim (1923), afirma que el individuo "no es verdaderamente él mismo, no realiza plenamente su naturaleza sino en la condición de sujetarse" (1992, p. 58). Es justamente esa "sujeción a la sociedad" lo que se nombra ordinariamente como socialización. En este mismo 
sentido, Giddens (1991, p. 93), sostiene que los adultos son "sujetos sociales" porque ellos son incorporados a la cultura del colectivo en el que nacen; para este autor, ellos obtienen esto "a través del proceso de socialización después de haber pasado poco a poco de la infancia a la edad adulta”. Aquí se citan, para comenzar, sólo estos dos ejemplos, sin embargo, es posible hallar, en toda la teoría sociológica, las más diversas definiciones del proceso de socialización, desde los clásicos hasta los contemporáneos.

En este sentido, a continuación se verá el camino que Dubar (2006a) ha franqueado para lograr su explicación sobre la socialización como "una articulación de dos procesos identitarios heterogéneos". Este autor presenta varias "configuraciones identitarias" como resultantes de una "doble transacción". Para él, las identidades son producidas por "la articulación entre una identidad atribuida por otro y la identidad de sí mismo, construida a través de la trayectoria anterior" (2006a, p. 186). Dubar explica así, cuatro "identidades sociales y profesionales típicas" contemporáneas.

Pretendiendo una doble ruptura con las definiciones durkheimianas -la inculcación de reglas o valores por las instituciones a unos individuos más o menos pasivos, y la representación lineal de la formación en término de acumulación de conocimientos-, Dubar comienza su exposición de las "grandes teorías" a partir de la Explicación en sociología de Piaget (1965). Como se sabe, este autor se interesa en la psicología y en el desarrollo mental del niño, más que en la sociología, pero sus contribuciones en la definición de la socialización son mayores ${ }^{6}$.

Sin embargo, es fácil estar de acuerdo con Dubar cuando afirma que el enfoque de Piaget sobre la socialización es restringido: "se centra únicamente sobre el individuo-niño e ignora, o minimiza las enormes variaciones que se pueden observar en los 'productos' de la socialización según las épocas, los tipos de sociedad, los medios sociales, los grupos o las clases" (Dubar, 2006a, p. 41). Por el contrario, el enfoque de los etnólogos y antropólogos sostiene que "la personalidad de los individuos es el producto de la cultura en la cual ellos han nacido" (p. 42). Para estos autores, la socialización se trata de "una incorporación progresiva de la cultura" donde se configura la "personalidad de base" (Kardiner, 1939).

No obstante, es la noción de "socialización anticipada" de Robert K. Merton, la que puede contribuir aquí. Al contrario de las teorías que se vienen de mencionar, su novedad consiste en la importancia de la proyección, y no solo sobre la recolección de los valores y las pautas de la comunidad de origen. La socialización anticipada es "el proceso por el cual un individuo aprende e interioriza los valores de un grupo (de referencia) al cual él desea pertenecer" (Dubar, 2006a, p. 61). Pero hay otra novedad aún más importante para los intereses de analizar la etapa de llegada de los nuevos trabajadores a la empresa: la socialización anticipada de Merton es un concepto que aplica a los adultos, y no a los niños.

Sin embargo, la debilidad de estas teorías salta a la vista, bien sea las "culturalistas" o las "estructural-funcionalistas", están determinadas por una visión holista. Muestran a un individuo socializado como "una suerte de autómata" y no como "un actor responsable" y autónomo. Además, este totalitarismo de las estructuras sociales se puede convertir en un obstáculo al "análisis sociológico de las conductas individuales en las sociedades modernas" (p. 65). Más adelante se verá que, al contrario de esta perspectiva de la socialización, el enfoque de la individuación procura resolver este totalitarismo estructural de una manera diferente.

Para continuar con el examen de las distintas definiciones de socialización, se observa que la noción de habitus puede servir para complementar las ideas anteriores. Dubar recuerda que es Bourdieu quien reconstruye esta noción, a la cual define como "sistemas de disposiciones duraderas y transferibles, estructuras estructuradas predispuestas a funcionar como estructuras estructurantes, es decir, en tanto que principios generadores y organizadores de prácticas y de representaciones" (citado por Dubar, 2006a, p. 69). Utilizando esta noción, Bourdieu se refiere a la socialización como "un proceso puramente social y casi mágico porque él asegura a la vez la adhesión subjetiva y la participación activa de los agentes a la reproducción de su posición social"

6. En este sentido de la socialización no se trata sin embargo, únicamente de Piaget. La influencia de los psicólogos en cuanto a la definición de la socialización es notable. Los sociólogos "se han apoyado mucho, frecuentemente sin gran creatividad teórica, sobre los estudios psicológicos (sobre todo los de Freud, Mead o Piaget) al momento de definir de manera más precisa los procesos psíquicos de formación del individuo" (Martuccelli, 2005, p. 2). Las cursivas son agregadas. 
(p. 70). Por supuesto, esta noción tendría que ser complementada con otra, teorizada también por Bourdieu, la idea de los campos sociales. Según él, un campo es "un espacio de juego" donde "unos agentes y unas instituciones están en lucha" para "apropiarse de provechos específicos" a la mejor manera de los mercados económicos.

Además, esta reciprocidad entre el habitus y el campo social, puede ser complementada con ciertas ideas de Habermas que se apoya en Hegel y recuerda la definición de identidad como el resultado de un reconocimiento recíproco: el "proceso de socialización concebido como exteriorización de lo subjetivo e interiorización de lo objetivo en la constitución del mundo social" (citado por Dubar, 2006a, p. 84). Sin embargo, la contribución que brinda Habermas para superar el enfoque de Piaget es que "la dialéctica motriz de la socialización" no está "entre el organismo y su medio" sino en "el vínculo entre el trabajo y la interacción". La "dialéctica del trabajo", ocupa, en la presentación de Habermas, una posición capital, porque no se trata únicamente de la acción instrumental. Para él "las identidades sociales y correlativamente, las formas de relaciones sociales en las cuales ellas se radican y se expresan, no pueden deducirse de sistemas de trabajo" ( $p$. 89 ), es preciso situarse en paralelo con el otro sistema que complementa esta dialéctica: "la acción comunicativa".

Sin embargo, hay que recordar que fue Mead, quien describió la socialización como "construcción de una identidad social en y por la interacción" con los otros. Mead complementa a Weber cuando el primero explica la socialización como una construcción progresiva de la comunicación del "sí mismo" (Self) con los otros miembros de la comunidad. Así se puede establecer, un puente entre el postulado de Mead con la célebre teoría de Berger y Luckmann: aunque se trate de la reproducción de la comunidad, no es una reproducción pasiva, sino una construcción de la realidad. Berger y Luckmann piensan que "la socialización nunca es completamente lograda", que no "es jamás total ni terminada" (1998, p. 174). Por ello proponen entonces su noción de "socialización secundaria".
Con este enfoque "comprensivo" de la socialización, Dubar comienza su propia síntesis en relación con las "grandes teorías". Él concluye presentando “cuatro 'modelos de socialización' en la articulación de los grandes tipos de acción (Weber), de los mecanismos de aprendizaje (Piaget), de las relaciones entre trayectorias y sistemas (Bourdieu), haciendo correspondencia a la hipótesis fundamental del dualismo social" (2006a, p. 104). Todo está listo para que este autor muestre su definición de identidad a partir de la articulación de "dos transacciones", la una interna, la otra externa, la una subjetiva, la otra objetiva, la una biográfica, la otra relacional, las cuales concluyen en una "identidad por sí" y en una "identidad por el otro", proceso éste que obedece a una dialéctica particular:

La construcción de las identidades se juega entonces más en la articulación entre los sistemas de acción proponiendo unas identidades virtuales y unas "trayectorias vividas"7 en el seno de las cuales se forjan las identidades "reales" las que se adhieren a los individuos. Ella puede analizarse también en términos tanto de continuidad entre identidad heredada e identidad vivida, como de ruptura, implicando ciertas conversiones subjetivas. También puede traducirse tanto por unos acuerdos como por unos desacuerdos entre la identidad "virtual", propuesta o impuesta por otro, e identidad "real" interiorizada o proyectada por el individuo (p. 112).

En cuanto al proceso identitario biográfico, Dubar recuerda que éste comienza desde la infancia, con las primeras identificaciones étnicas, de sexo, de clase, etc., pero que entre esta identidad "heredada" y la identidad "escolar", emerge "un campo de lo posible en el cual se despliegan, desde la infancia a la adolescencia, y a todo lo largo de la vida, todas nuestras estrategias identitarias" (p. 116). Además de la escuela elemental, este autor llama la atención sobre la legitimidad de dos variables fundamentales en nuestra contemporaneidad que son el trabajo y la formación:

Si los modos de construcción de las categorías sociales a partir de los campos escolar y profesional han adquirido una tal

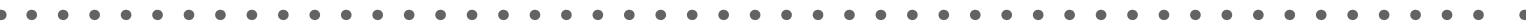

7. Nota de Dubar: La noción de "trayectoria vivida" designa la manera por la cual los individuos reconstruyen subjetivamente los eventos que ellos juzgan significativos de su biografía. Estas trayectorias pueden ser aprendidas a través de sus discursos implicando unas categorizaciones y unas argumentaciones específicas (Demazière y Dubar, 1997). 
legitimidad, es más porque las esferas del trabajo y del empleo [...], pero también la de la formación [...] constituyen unos dominios pertinentes de las identificaciones sociales de los individuos mismos (p. 116).

En cuanto al proceso identitario relacional, retomando a Sainsaulieu, Dubar le brinda prioridad al espacio de reconocimiento identitario, porque, según el primero, la identidad "es menos un proceso biográfico de construcción de sí que un proceso relacional de inversión de sí mismo" (citado por Dubar, 2006a, p. 119). Sin ánimo de exhaustividad, se invita, entonces a observar los cuatro procesos de configuración identitaria que Dubar desarrolla en su matriz, los cuales pueden representarse en los siguientes ejemplos (Tabla 2):

La exclusión (externa), se evidencia en la identidad (virtual) que es atribuida por los demás; es decir, no es la identidad creada por el individuo (por ejemplo, la identidad que atribuye la dirección de la gran empresa a sus obreros: que son poco capacitados, de bajo perfil, burdos, etc.).

El bloqueo (interno) a la identidad, puede observarse cuando la identidad (virtual) es creada por el individuo a través de su propia trayectoria anterior y no se ajusta a las nuevas exigencias (por ejemplo cuando se modifica la organización del trabajo e irrumpe, cada vez con mayor potencia, la necesidad del trabajador a ser polivalente; cuando la dirección pretende que se pase de obrero ejecutor de tareas al empleado "gerente" de su puesto de trabajo).

La promoción (interna), genera un tipo de identidad que está determinada por lo que se conoce como el modelo de carrera interna: cuando ha primado la estabilidad laboral (el estado de bienestar, el paternalismo, etc.) y la movilidad se da al interior de la empresa (tanto las promociones como los estancamientos).

La conversión (externa) de la identidad, se puede observar en las últimas generaciones; después de las modificaciones estructurales de la organización del trabajo: la evaluación individualizada del desempeño, la "virtualización" de las relaciones de trabajo, etc. (se pasa de la identidad por afiliación al otro, a la identidad por competencia con el otro).

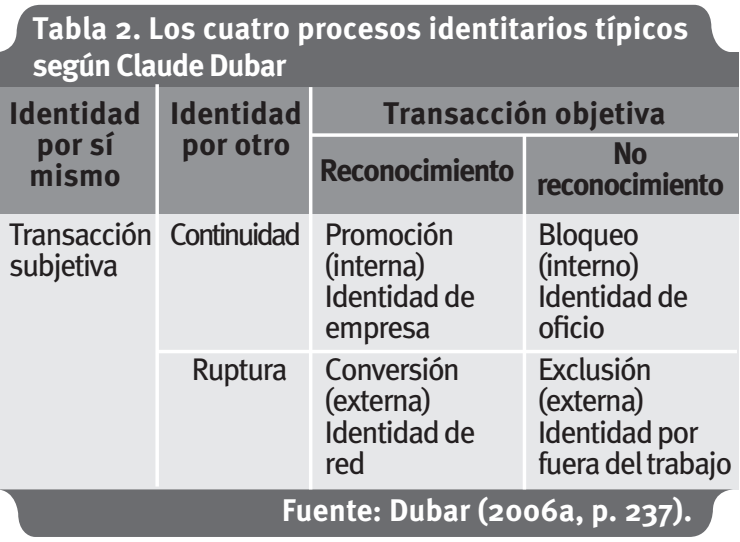

Sin embargo, hay que recordar que hasta aquí se ha seguido a Dubar, no exactamente para utilizar sus esquemas de los procesos identitarios típicos -los cuales pueden, sin duda, resultar muy útiles también para los especialistas en gestión humana-, sino para examinar el desarrollo del concepto de socialización y ciertos recursos epistemológicos para establecer una comparación entre la etapa de llegada de los nuevos trabajadores y la socialización definida como proceso.

\section{Otra estrategia de análisis: la individuación}

Ya se indicó que es posible examinar la etapa de llegada de los nuevos trabajadores a la empresa a partir de otra estrategia de análisis sociológico denominada la individuación. Este enfoque es completamente diferente al de la socialización; es la perspectiva utilizada por ciertas "sociologías del individuo". Pero ¿por qué utilizar una sociología del individuo? En un apartado anterior de este mismo artículo se señaló "la perspectiva de la empresa". En tal perspectiva es evidente que la organización -como sistema organizado de relaciones sociales- determina y regula las conductas de los individuos, por eso, dentro de esa lógica, la única alternativa es la de socializar las vivencias individuales.

Sobre cualquier tipo de sociología que tome apoyo la teoría de la organización, la empresa es comprendida como una estructura más o menos organizada. Inclusive, no se puede olvidar que la sociología, desde las épocas de los "padres fundadores" (incluso desde sus "precursores"), emerge a partir del interés de explicar las grandes estructuras sociales: Marx, Durkheim, o Weber (Tönnies, Simmel, Pareto, etc.), cada uno, a su manera, tenía esa pretensión. La sociología es justamente eso: la explicación del conjunto de fuerzas sociales. "La verdadera unidad disciplinar de la sociología, más allá de las escuelas y 
de las teorías -recuerda Martuccelli- viene de ese proyecto de comprender las experiencias personales a partir de sistemas organizados de relaciones sociales":

Todos los enfoques, a pesar de su diversidad, comulgan alrededor de ese modelo general, que hace de la posición del actor el mejor operador analítico para dar cuenta de sus maneras de ver, de actuar y de percibir el mundo. No tiene nada de sorprendente entonces que en el seno de ese modelo, el proceso de socialización juegue un rol preponderante (2009, p. 16).

Por eso, hay que complementar este enfoque con el de una sociología del individuo actualizada. Se precisa una reorganización teórica y metodológica para estudiar los problemas sociológicos contemporáneos, como el de la llegada de los nuevos trabajadores a la empresa. Esta es la invitación que se hace a los especialistas de la gestión humana: "eso que debe modificarse -insiste Martuccelli- es la voluntad de comprender exclusivamente los individuos a partir de una estrategia que brinda un rol interpretativo dominante a las posiciones sociales" (2009, p. 17). Se trata de separarse de lo que Eugène Enriquez llama "el triunfo de las teorías que pregonan, incluso sin decirlo, un determinismo absoluto de los procesos sociales" (2003, p. 118). Es necesario entonces, buscar otra perspectiva que sobrepase esa reducción ontológica.

Sin embargo, hay que hacer por ahora tres advertencias. La primera es que no se está proponiendo la simple aplicación del "individualismo metodológico" (Boudon, 1979); la acción intencional no es aquí privilegiada. La segunda, es que no se trata tampoco del individualismo de los economistas clásicos: no se propone, en absoluto, partir de la idea del individuo como egoísta racional. La tercera, es que este individualismo no es la psicologización de los problemas sociológicos. En resumen, la sociología que se propone aquí, considera que el individuo no es solamente un "actor social". Por supuesto que es un actor empírico, pero también, y en simultánea, es un ser moral-histórico (Sartre, 1985a; Dumont, 1983; Castoriadis, 1975).

Se trata, mejor, de una sociología que interroga la experiencia personal, pero en el contexto de una contemporaneidad compleja e histórica. Es una sociología que da cuenta de la dualidad individuo-sociedad (Elias, 1991; Enriquez, 2003; Sévigny, 2009; Rhéaume, 2009a, 2009b); una sociología cuya pretensión es comprender la configuración de la "sociedad de los individuos" actual:

La orientación principal de [la] postura de investigación [...] es una de comprehensión, de religar directamente lo vivido, la experiencia personal y la sociedad global, bajo la forma de cuestionamiento, de explorar cómo, cada uno o cada una, supera e integra la dualidad individuo y sociedad (Rhéaume, 2009a, p. 6).

Así, la individuación como estrategia de análisis sociológico estaría más bien ligada a la emergencia de un "nuevo individualismo institucional” (Beck, 1986). Está fundada, se insiste, sobre una sociología epistemológicamente renovada ${ }^{8}$. Nótese la pertinencia de este tipo de concepción sociológica para examinar los problemas organizacionales, como la etapa de llegada de los nuevos trabajadores a la empresa. Para examinar este enfoque, ya se advirtió, se ha tomado apoyo sobre todo en la teorización que viene desarrollando Martuccelli. Se insiste que este autor, explica justamente, por qué la unidad de la sociología, como disciplina, viene de comprender lo individual como consecuencia de lo social:

Los sociólogos durante mucho tiempo han repugnado interesarse en el individuo, porque ellos consideraban, en el fondo, que ese nivel de análisis no era realmente el suyo, $y$, sobre todo, que cuando esto era objeto de estudio (porque efectivamente sí lo ha sido en el pasado), no había sino un interés secundario, gracias a que era el reverso de las estructuras sociales (de lo social interiorizado o un soporte de las estructuras). Hay que reconocer que en

8 Sinduda la je filosóficamente el concepto de individuación antes de pretender desagregarlo en operadores analíticos para la investigación de campo; sin embargo, se advierte que estas ideas no alcanzan a ser revisadas en el presente ejercicio. 
los trabajos actuales, la mirada se pone cada vez más sobre el propio individuo. Digamos rápidamente que, a la exclusividad de una visión descendente (de la sociedad al individuo) se añade, aunque no se opone, una visión ascendente (del individuo hacia la sociedad) (Martuccelli, 2005, p. 1)9.

El enfoque de la individuación es precisamente eso: una forma de buscar este equilibrio metodológico para estudiar la sociedad de los individuos contemporáneos. Sin embargo, para comprender la individuación como estrategia de análisis sociológico, es importante, primero, diferenciar entre la individuación y la individualización. En este contexto, la individualización es "la interpretación de un proceso específico a la segunda modernidad, que después de una serie de cambios institucionales, forja los individuos aumentando sus capacidades de reflexión" (Martuccelli, 2009, p. 23). Ya se dijo que según Beck (1986), la tesis de la individualización es inseparable de "la emergencia de un nuevo individualismo institucionalizado". Por su parte, la individuación "designa un proceso más amplio, porque otros factores estructurales, y no sólo el trabajo de las instituciones, en el sentido fuerte del término, son tomados en cuenta". La dinámica de la individuación "se esfuerza así de interpretar en el horizonte de una vida -o de una generación- las grandes transformaciones históricas de una sociedad" (Martuccelli, 2009, pp. 23-24).

Entonces, para preguntarse por la individuación como interrogación sobre el tipo de individuo que fabrica estructuralmente una sociedad, hay que examinar el proceso de la individualización a partir de un operador metodológico particular: las "pruebas". Aquí no importa la posición del actor como operador analítico, no se busca su identificación como instrumento para "fijarlo" en procura de comprenderlo. No es determinante la localización (topológica) al interior de un grupo, de la cultura o de la profesión para explicar al individuo. No se privilegia la interiorización de reglas y valores ni se utiliza la lógica de los sistemas, de los campos o de las configuraciones (Parsons; Bourdieu; Elias). En resumen, no se trata de socialización. Este enfoque no trabaja con el individuo socializado, al contrario, metodológicamente trabaja con "el individuo individualizado" (Singly, 2003).

En el enfoque de la individuación, tal como se acaba de indicar, la "prueba" es el operador analítico. Aquí, es el propio individuo el que muestra sus tensiones según cada momento de la experiencia biográfica; aquí, él está "obligado a circular en una ambivalencia irreducible" (Martuccelli, 2009, p. 23). Por eso, un operador analítico como la prueba, debe ser inseparable de herramientas heurísticas de tipo biográfico como la "novela familiar"10, o las célebres "historias de vida". Martuccelli propone entonces los "retratos":

Con los retratos individuales, se trata de establecer un espacio sui generis de análisis, susceptible de dar cuenta del trabajo sobre sí mismo que cada actor cumple, con el fin de fabricarse en tanto que sujeto en medio de ecologías sociales cada vez más personalizadas (p. 27).

Sin embargo, hay que insistir que estos retratos no son, en absoluto, para mostrar que la experiencia individual reproduce simplemente las grandes tendencias estructurales. Es preciso escapar a la tentación fuerte de "regresar" sobre la idea del personaje social, sobre las ambiciones explicativas totalizadoras, sobre la existencia de modelostipos psicologizantes, y sobre la ilusión del orden establecido o por establecer. En este sentido, no es suficiente con la aplicación de etnometodologías o sociologías fenomenológicas preconcebidas, sino, justamente, la actualización de las existentes y la construcción de unas nuevas ${ }^{11}$. La Tabla 3 resume el paralelo propuesto hasta ahora.

9. Cursivas agregadas.

10. Se sabe que Freud propone su "Novela familiar del neurótico" desde 1908. Sin embargo, son los seguidores francófonos de la llamada "Sociología clínica" quienes, utilizando este nombre (Roman familial) han actualizado y desarrollado una teorización tendiente a la aplicación de la biografía, como instrumento metodológico (Enriquez, 2009, 2003; Gaulejac, 1999; Enriquez et al., 1993).

11. Recuérdese el ejercicio (comenzado en 1957, e inacabado) de Jean-Paul Sartre procurando mostrar a partir de un combate de boxeo la encarnación individual y la totalización del conjunto social (Sartre, 1985b). 


\section{El enfoque de la socialización}

La posición del actor es tomada como operador analítico Se busca su identificación para "fijarlo" y comprenderlo. Interesa la localización (topológica) al interior de un grupo, de la cultura o de la profesión para explicar al individuo.

Es necesario examinar su interiorización de reglas y valores.

Aparecen unos espacios sociales que determinan su conducta o sus proyecciones.

Se utiliza la lógica de los sistemas, de campos o de configuraciones (Parsons; Bourdieu; Elias).

Este enfoque trabaja con el individuo socializado.

\section{El enfoque de la individuación}

La "prueba" es tomada como operador analítico.

El propio individuo muestra sus tensiones según cada momento de la experiencia biográfica. Está “obligado a circular en una ambivalencia irreducible" (Martuccelli, 2009, p. 23).

Es portador de un imaginario actualizado de reglas y valores.

Su propio relato biográfico le determina como individuo.

Los "retratos" son la herramienta heurística.

Este enfoque trabaja con el individuo individualizado. Fuente: Bermúdez, H. L. (2012).
Ese es uno de los objetivos de este artículo. No se quiere invitar a arriesgar un trabajo empírico sin la suficiente decantación teórica, porque la mencionada “tentación de regresar" es, en este caso, una inercia consecuente de la enorme acumulación epistemológica unidireccional del management en general y de la gestión humana en particular. En pocas palabras, la propuesta es blindarse teóricamente, bien sea contra la ingenuidad académica, o contra la tranquilidad que procuran ciertas tradiciones epistemológicas ya consolidadas, y que, a nuestro juicio, necesitan cotejarse y criticarse.

\section{Conclusiones}

El comienzo de las labores de un nuevo trabajador en la empresa implica una experiencia biográfica muy significativa. La etapa de llegada al colectivo organizacional, como problema de estudio, brinda una oportunidad académica extraordinaria: aquella de buscar explicaciones suplementarias.

En ninguno de los textos especializados de gestión humana examinados en el presente estudio, pudo hallarse una definición de la inducción general diferente a la de una propuesta de adaptación del trabajador por la vía de lo que tales textos llaman "socialización organizacional”.

Los elementos teóricos y metodológicos con los cuales la tradición epistemológica dominante de la gestión humana ha procurado explicar la etapa de llegada de los nuevos trabajadores como una socialización, son incapaces de dar cuenta de muchas complejidades inherentes a esta etapa. Se requieren otros instrumentos conceptuales diferentes a los que hasta ahora se han utilizado.
Ese ha sido, justamente, el propósito de este artículo: poner a disposición de los estudiosos, del management y de la gestión humana, algunos elementos cuya fortaleza teórica y metodológica ha sido probada con suficiente investigación empírica en problemas sociológicos diversos (Sévigny, 2009; Rhéaume, 2009b; Martuccelli, 2006).

Tales recursos, han sido en general, desconocidos por la tradición epistemológica de la gestión humana. Resta pues -y aquí se deja el reto planteado a los colegas investigadores-, ir al trabajo de terreno con un problema como éste, ya mejor delimitado y acudir a un arsenal conceptual decididamente renovado.

Porque mientras se sigan examinando las dinámicas humanas con herramientas teóricas incompletas o en desuso, no solo se estará lejos de la comprensión de las mismas, sino que se incurre en el peligro de aportar a la perpetuación y el desarrollo de unas prácticas administrativas que reproducen tal incomprensión y tal desconocimiento sobre los aconteceres de los seres humanos en los escenarios laborales contemporáneos.

\section{Referencias}

Aktouf, 0. (2008). Halte au gâchis. En finir avec l'économie-management à l'américaine. Montréal, Canadá: Liber.

Aktouf, 0. (2002). La stratégie de l'autruche. Montréal, Canadá: Écosociété.

Almacenes ÉXITO. (2001). Normalización del proceso de inducción general. Documento interno de trabajo. Vicepresidencia de Gestión Humana. Envigado, Colombia: Grupo Éxito. 
Bargues, E. (2008). Gérer la socialisation organisationnelle pour développer-maintenir la culture de l'organisation: vers un enrichissement des connaissances des pratiques. Actes XIXe Congrès AGRH. Dakar, Senegal, 9-12/11/2008. AGRH.

Bargues, E. (2006). Pratiques de socialisation et attitudes au travail des nouveaux dans les TPE. Actes XVII Congrès AGRH, Reims, Francia, 24-26/11/2006. AGRH.

Barnard, C. (1938). The Functions of the Executive. Cambridge, USA: Harvard University Press.

Bayad, M., Arcand, G., Arcand, M. y Allani, N. (2004). Gestion stratégique des ressources humaines: Fondements et modèles. Revue internationale des relations de travail, 2(1) (pp. 74-93).

Beck, U. (1986). La société du risque. Paris, Francia: Aubier.

Berger, P. y Luckmann, T. (1998). La construcción social de la realidad. Buenos Aires, Argentina: Amorrortu.

Bermúdez, H. (2010). ¿Es posible una gestión humana no funcionalista? Descripción de un modelo estratégico de gestión de personal. Universidad y Empresa, (18) (pp. 174-202).

Bermúdez, H. (2009). La gestión humana estratégica: Hacia la búsqueda de su coherencia humanista. Economía gestión y desarrollo, (7) (pp. 149-168).

Bonny, Y. (2004). Sociologie du temps présent. Modernité avancée ou postmodernité? Paris, Francia: Armand Colin.

Bonny, Y. (1998). Modernité avancée ou postmodernité? Enjeux et controverses. Société, (18/19) (pp. 87-121).

Boudon, R. (1979). La logique du social. Paris, Francia: Hachette.

Boussaguet, S. (2007). Réussir son entrée dans l'entreprise: le processus de socialisation organisationnelle du repreneur. Économies et sociétés, 41(1) (pp. 145-163).

Boussaguet, S. (2005). L'entrée dans l'entreprise du repreneur: un processus de socialisation repreneuriale. Tesis doctoral. Programa Doctoral en Ciencias de la Gestión, Université Montpellier I, Montpellier, Francia.

Bustamante, A. y Bermúdez, H. (2010). La motivación de los empleados subcontratados. Una paradoja incesante. $A D$-minister, (17) (pp. 35-57).

Bustamante, A. (2006). Algunas implicaciones de la subcontratación en la motivación de los trabajadores contratados bajo esta modalidad. Memoria de grado. Maestría. Programa de Maestría en Ciencias de la Administración, Universidad EAFIT. Medellín, Colombia [no publicada].

Cane, S. (1997). Cómo triunfar a través de las personas. Bogotá, Colombia: McGraw-Hill.

Cascio, W., Thacker, J., Blais, R. (1999). La gestion des ressources humaines. Montréal, Canadá: Chenelière/McGraw-Hill.

Castel, R. (1997). Les métamorphoses de la question sociale. Une chronique du salariat. Paris, Francia: Fayard.

Castoriadis, C. (1975). L'institution imaginaire de la société. Paris, Francia: Seuil.

Conninck, F., Guillot, C. (2007). L'individualisation du rapport au temps, marqueur d'une évolution sociale. ¿Interrogations?, (5) (pp. 22-42). Recuperado 21/03/2011 de: http://www.revue-interrogations.org/index.php?num_ID $=8$

Conninck, F. (2006). A distance de soi-même. L'individu clivé vu par Max Weber et Georg Simmel et son intérêt heuristique actuel. ¿Interrogations?, (2) (pp. 20-39). Recuperado 21/03/2011 de: http://www.revue-interrogations.org/ index.php?num_ID=3

Chiavenato, I. (2002). Gestión del Talento Humano. México DF, México: McGraw-Hill.

Crozier, M., Friedberg, E. (1977). L'acteur et le système. Paris, Francia: Seuil.

Dolan, S., Schuler, R. y Valle, R. (1999). La gestión de los Recursos Humanos. Madrid, España: McGraw-Hill.

Dubar, C. (2007). La crise des identités. L'interprétation d'une mutation. Paris, Francia: Puf. 
Dubar, C. (2006a). La socialisation. Paris, Francia: Armand Colin.

Dubar, C. (2006b). Faire de la sociologie. Un parcours d'enquêtes. Paris, Francia: Belin.

Dumont, L. (1983). Essais sur l'individualisme. Une perspective anthropologique sur l'idéologie moderne. Paris, Francia: Seuil.

Durkheim, E. (1992). L'éducation morale. Paris, Francia: Puf.

Ehrenberg, A. (1998). La fatigue d'être soi. Dépression et société. Paris, Francia: Odile Jacob.

Ehrenberg, A. (1995). L'individu incertain. Paris, Francia: Calmann-Lévy.

Elias, N. (1991). Qu'est-ce que la sociologie ? La Tour d'Aigues, Francia: Éditions de l'Aube.

Enriquez, E. (2009). Le pouvoir, l'État et le sujet dans le monde actuel. Sociologie et sociétés, 41(1) (pp. 159-176).

Enriquez, E. (2003). L'organisation en analyse. Paris, Francia: Puf.

Enriquez, E., Houle, G., Rhéaume, J., Sévigny R. (Dir.). (1993). L'analyse clinique dans les sciences humaines. Montréal, Canadá: Saint-Marin.

Gauchet, M. (1998). Essai de psychologie contemporaine I. Un nouvel âge de la personnalité. Le Débat, (99) (pp. 164-181).

Gaulejac, V. de (2005). La société malade de la gestion. Paris, Francia: Seuil.

Gaulejac, V. de (1999). L'histoire en héritage. Roman familial et trajectoire sociale. Paris, Francia: Desclée de Brouwer.

Giddens, A. (1993). Une théorie critique de la modernité avancée. En: Audet, M. \& Bouchikhi, H. (Dir.) Structuration du social et modernité avancée. Québec, Canadá: Les Presses de l'Université Laval (pp. 29-53).

Giddens, A. (1991). Sociología. Madrid, España: Alianza.

Gómez-Mejía, L., Balkin, D., y Cardy, R. (1999). Gestión de recursos humanos. Madrid, España: Prentice May.
Homero (tr.1947). L’Odyssée. Tr. Francés. M. Dufour y J. Raison. Paris, Francia: Flammarion.

Kardiner, A. (1939). The Individual and his Society. New York, USA: Columbia University Press.

Koontz, H., y Weihrich, H. (1994). Administración una perspectiva global. México DF, México: McGraw-Hill.

Lipovetsky, G. (2004). Les Temps hypermodernes. Paris, Francia: Grasset y Fasquelle.

Martuccelli, D. (2009). Qu'est-ce qu'une sociologie de l'individu moderne? Pour quoi, pour qui, comment? Sociologie et sociétés, 41(1) (pp. 15-33).

Martuccelli, D. (2006). Forgé par l'épreuve. L'individu dans la France contemporaine. Paris, Francia: Armand Colin.

Martuccelli, D. (2005). Les trois voies de l'individu sociologique. Recuperado 11/04/2011 de: http://espacestemps.net/document1414.html

Martuccelli, D. (2002). Introduction. En: Martuccelli, D. Grammaires de l'individu. Paris, Francia: Gallimard (pp. 11-42).

Notais, A. (2010). Mobilité interne et socialisation organisationnelle: Apprentissage et identité en question. Actes XXI Congrès AGRH. Rennes/ Saint-Malo, Francia, 17-19/11/2010. AGRH.

Notais, A. (2009). Une approche subjective de la mobilité interne par la socialisation. Actes $X X$ Congrès AGRH. Toulouse, Francia, 2021/11/2009. AGRH.

Perrot, S. (2009). Échelles de mesure de la socialisation organisationnelle: état de l'art et perspectives. Management international, 13(4) (pp. 115-127).

Perrot, S. (2008). Évolution du niveau de socialisation organisationnelle selon l'ancienneté: une analyse des premiers mois dans l'entreprise. Management international, 11(3) (pp. 231-258).

Rhéaume, J. (2009a). Présentation. Sociologie et sociétés, 41(1), (pp. 5-13).

Rhéaume, J. (2009b). La sociologie clinique comme pratique de recherche en institution. 
Le cas d'un centre de santé et services sociaux. Sociologie et sociétés, 41(1) (pp. 195-215).

Riordan, C., Weatherly, E., Vandenberg, R. \& Self, R. (2001). The effect of pre-entry experiences and socialization tactics on newcomer attitudes and turnover. Journal of Managerial Issues, 13(2) (pp. 159-176).

Roethlisberger, F. \& Dickson, W. (1976 [1939]). Management and the Worker. Cambridge, USA: Harvard University Press.

Sartre, J.P. (1985a). Le conflit, moment d'une totalisation ou déchirure irréductible? En: Sartre, J.P. Critique de la raison dialectique, tome II. Paris, Francia: Gallimard (pp. 11-25).

Sartre, J.P. (1985b). Rapports du conflit, singulier avec les conflits fondamentaux de l'ensemble social. En: Sartre J.P., Critique de la raison dialectique, tome II. Paris, Francia: Gallimard (pp. 26-60).

Schein, E. (1982). La socialización organizacional y la profesión de la administración de empresas. En: Kolb, D. Psicología de las organizaciones: Problemas contemporáneos (sp.). Madrid, España: Prentice-Hall.

Schein, E. (1971). The individual, the organization, and the career: A conceptual scheme. The Journal of Applied Behavioural Science, 7(4) (pp. 401426).

Schein, E. (1968). Organization socialization and the profession of management. Industrial Management Review, 9 (2) (pp. 1-16).

Sekiou, L., Blondin, L., Fabi, B., Bayad, M., Peretti, J., Alis, D., et al. (2001). Gestion et mobilisation des ressources humaines: une perspective internationale. Montréal, Canadá: Éditions 4L Inc.

Sévigny, R. (2009). Sociologie clinique et schizophrénie en Chine post-maoïste: l'expérience de Lu Lu. Sociologie et sociétés, 41(1) (pp. 125-158).
Simondon, G. (2009). La individuación a la luz de las nociones de forma y de información. Buenos Aires, Argentina: Cactus y La Cebra.

Simmel, G. (1989). Philosophie de la modernité. Paris, Francia: Payot.

Singly, F. (2003). Les uns avec les autres. Paris, Francia: Armand Colin.

Soussi, S. (2009). Sociologie du travail. Colloque fermé aux étudiants inscrits dans le cours doctoral SOC-8712. Montréal, Canadá. 03/12. Département de Sociologie. Université du Québec à Montréal.

St-Onge, S., Audet, M., Haines, V., Petit, A. (2004). Relever les défis de la gestion des ressources humaines. Montréal, Canadá: Gaëtan Morin.

Van Maanen, J. (1976). Socialization to work. En: Dubin, R. (ed.) Handbook of work, organization and society. Chicago, USA: Rand-McNally (pp. 67-130).

Van Maanen, J. \& Schein, E. (1979). Toward a theory of organizational socialization. Research in Organizational Behaviour, 1(1) (pp. 209-264).

Weber, M. (2002). L'éthique protestante et l'esprit du capitalisme. Paris, Francia: Flammarion.

Weber, M. (1965). Essai sur quelques catégories de la sociologie compréhensive. En: Weber, M. Essais sur la théorie de la science. Paris, Francia: Plon (pp. 325-398).

Wils, T., Labelle, G., Guérin, G. (1989). La gestion stratégique des ressources humaines: Un reniement du rôle social de l'entreprise? Relations Industrielles, 44(2) (pp. 354-374). 